\title{
MEAN EXIT TIME OF A DIFFUSION PROCESS FROM A SMALL SPHERE
}

\author{
MARK A. PINSKY ${ }^{1}$
}

\begin{abstract}
The first two nonzero terms in the asymptotic expansion of the mean exit time from a small sphere are expressed in terms of the radius, the scalar curvature of the Riemannian metric and the vector field which defines the drift in the infinitesimal generator.
\end{abstract}

Consider a nondegenerate diffusion process in $R^{n}$. Let $T_{\varepsilon}$ be the exit time from a ball of radius $\varepsilon$, where we use the Riemannian distance determined by the second order coefficients. In this note we compute the first two nonzero terms in the asymptotic expansion of $E_{m}\left(T_{\varepsilon}\right), \varepsilon \downarrow 0$. We have the following result:

THEOREM. When $\varepsilon \downarrow 0$

$$
E_{m}\left(T_{\varepsilon}\right)=\frac{\varepsilon^{2}}{2 n}+\frac{\varepsilon^{4}}{12 n^{2}(n+2)}\left(\tau_{m}-3(\operatorname{div} b)_{m}-\frac{3}{2}|b|_{m}^{2}\right)+O\left(\varepsilon^{6}\right),
$$

where $\tau_{m}$ is the scalar curvature of the Riemannian metric $\left(g_{i j}\right)$ and $\left(b_{i}\right)$ is the vector field which appears when the infinitesimal generator is written as $L=\Delta+b \cdot \nabla$, or in coordinate form

$$
L=\sum_{i j} \frac{1}{\sqrt{g}} \frac{\partial}{\partial x_{i}}\left(\sqrt{g} g^{i j} \frac{\partial}{\partial x_{i}}\right)+\sum_{i} b_{i} \frac{\partial}{\partial x_{i}}
$$

with $g^{i j}=\left(g^{-1}\right)_{i j}$ and $g=\operatorname{det}\left(g_{i j}\right)$.

Proof of THE Theorem. We use the perturbation method initiated in [1]. In a system of normal coordinates $\left(x_{1}, \ldots, x_{n}\right)$ at $m \in \mathbf{R}^{n}$ we have

$$
\Delta=\Delta_{-2}+\Delta_{0}+\cdots, \quad b \cdot \nabla=B_{-1}+B_{0}+\cdots,
$$

where

$$
\begin{aligned}
& \Delta_{-2}=\sum_{i} \frac{\partial^{2}}{\partial x_{i}^{2}}, \\
& \Delta_{0}=\frac{1}{3} \sum_{i a j b} R_{i a j b} x_{a} x_{b} \frac{\partial^{2}}{\partial x_{i} \partial x_{j}}-\frac{2}{3} \sum_{i a} \rho_{i a} x_{a} \frac{\partial}{\partial x_{i}}, \\
& B_{-1}=\sum_{i} b_{i} \frac{\partial}{\partial x_{i}}, \quad B_{0}=\sum_{i j} b_{i j} x_{j} \frac{\partial}{\partial x_{i}}
\end{aligned}
$$

Received by the editors March 1, 1984.

1980 Mathematics Subject Classification. Primary 58G32, $60 \mathrm{~J} 60$.

Key words and phrases. Mean exit time, diffusion process.

${ }^{1}$ Research supported by National Science Foundation Grant MCS-8403154. 
and $\left(R_{i a j b}\right)$ is the curvature tensor at $m \in \mathbf{R}^{n}, \rho_{i a}=\sum_{j} R_{j i j a}$ is the Ricci tensor and $b_{i}=b_{i}(m), b_{i j}=\partial b_{i}(m) / \partial x_{j}$. The mean exit time is the solution of $L f=-1$ which vanishes on the $\varepsilon$-sphere. We obtain an approximate solution in the form $\bar{f}_{\varepsilon}(x)=$ $\varepsilon^{2} f_{0}(x / \varepsilon)+\varepsilon^{3} f_{1}(x / \varepsilon)+\varepsilon^{4} f_{2}(x / \varepsilon)$, where

$$
\begin{aligned}
& \Delta_{-2} f_{0}+1=0, \\
& \Delta_{-2} f_{1}+B_{-1} f_{0}=0, \\
& \Delta_{-2} f_{2}+B_{-1} f_{1}+\left(\Delta_{0}+B_{0}\right) f_{0}=0
\end{aligned}
$$

and the functions $f_{0}, f_{1}, f_{2}$ are required to be zero on the unit sphere. Solving explicitly, we have

$$
\begin{aligned}
& f_{0}=\frac{1-|x|^{2}}{2 n}, \quad f_{1}=\frac{1}{2 n(n+2)}\left(1-|x|^{2}\right) \sum_{i} b_{i} x_{i} \\
& B_{-1} f_{1}+B_{0} f_{0}=\frac{\left(\sum b_{i} x_{i}\right)^{2}}{n(n+2)}-\frac{\left(1-|x|^{2}\right)}{2 n(n+2)}|b|^{2}-\frac{1}{n}\left(\sum_{i j} b_{i j} x_{i} x_{j}\right) \\
& \Delta_{0} f_{0}=-\frac{1}{3 n} \sum_{i j} \rho_{i j} x_{i} x_{j} .
\end{aligned}
$$

Applying Lemma 6.3 of [1], we have

$$
\begin{aligned}
f_{2}(0)= & \frac{\tau_{m}}{12 n^{2}(n+2)}+\frac{1}{n(n+2)} \frac{|b|_{m}^{2}}{4(n+2) n} \\
& -\frac{|b|_{m}^{2}}{2 n(n+2)}\left[\frac{1}{2 n}-\frac{1}{4(n+2)}\right]-\frac{(\operatorname{div} b)_{m}}{4 n^{2}(n+2)} \\
= & \frac{1}{12 n^{2}(n+2)}\left(\tau_{m}-3(\operatorname{div} b)_{m}-\frac{3}{2}|b|_{m}^{2}\right)
\end{aligned}
$$

The proof is complete.

\section{REFERENCES}

1. A. Gray and M. Pinsky, The mean exit time from a small geodesic ball in a Riemannian manifold, Bull. Sci. Math. (2) 107 (1983), 345-370.

DePartment of MATHEMatics, NORThWESTERn University, EVANSTON, Illinois 60201 